\title{
Pulmonary Sarcoidosis That Developed During the Treatment of a Patient With Crohn Disease by Using Infliximab
}

\author{
Tae Kyun Kim, Sun Hyung Kang, Hee Seok Moon, Jae Kyu Sung, Hyun Yong Jeong, Hyuk Soo Eun \\ Division of Gastroenterology, Department of Internal Medicine, Chungnam National University College of Medicine, Daejeon, Korea
}

For inflammatory bowel disease (IBD), antitumor necrosis factor treatment offers a new direction for both patients and medical doctors. This treatment has dramatically improved the quality of life for patients with ulcerative colitis and Crohn disease (CD). However, with increasing usage and longer follow-up periods, a wider range of possible adverse effects may be encountered. We report an unusual case of pulmonary sarcoidosis developed during the treatment of a patient with CD by using infliximab. A 30-year-old male who had been treated for CD with infliximab for 18 months was admitted due to abnormal opacities on chest radiography. Chest computed tomography displayed clustered small nodules in both lobes and enlarged multiple lymph nodes. The patient was diagnosed with sarcoidosis from the results of a biopsy of the subcarinal lymph node. Lung lesions were improved five months after infliximab was stopped.

Keywords: Crohn disease; Tumor necrosis factor-alpha; Sarcoidosis; Adverse event

\section{INTRODUCTION}

Infliximab, one of the anti-tumor necrosis factor (TNF)-a agents, has recently been applied to treat patients with rheumatoid arthritis (RA) and other diseases, such as inflammatory bowel disease (IBD) and psoriasis. In addition, anti-TNF agents have been reported to be effective for the treatment of refractory sarcoidosis, which does not respond to treatment with steroids or immunosuppressive drugs. However, with increasing usage and longer follow-up periods, a wider range of possible adverse effects may emerge. A few reports have been published of cases where patients with RA presented with sarcoidosis after anti-TNF treatment. However, few reports have been published of patients with IBD who developed sarcoidosis after this treatment [1]. The pres-

Received: August 8, 2016 - Accepted: November 8, 2016

Correspondence to: Hee Seok Moon, M.D.

Division of Gastroenterology, Department of Internal Medicine, Chungnam National, University School of Medicine, 282 Munhwa-ro, Jung-gu, Daejeon 35015, Korea

Tel: +82-42-280-8049, Fax: +82-42-257-5753, E-mail: mhs1357@cnuh.co.kr

(c) 2017 The Korean Society of Coloproctology

This is an open-access article distributed under the terms of the Creative Commons Attribution NonCommercial License (http://creativecommons.org/licenses/by-nc/4.0) which permits unrestricted noncommercial use, distribution, and reproduction in any medium, provided the original work is properly cited. ent case study describes a patient with IBD who received infliximab treatment and was also found to have sarcoidosis.

\section{CASE REPORT}

In 2006, a 22-year-old man was diagnosed with Crohn disease (CD). He first came to our unit 4 years later. At that time, he had stopped taking the medicine independently and presented with gastrointestinal symptoms. Mesalazine and azathioprine were initiated in 2010 at an age 26 years. After 2 years of treatments, the patient's gastrointestinal symptoms were uncontrolled, and an examination by colonoscopy revealed strictures in the descending colon (Fig. 1). Due to worsening CD, treatment with an antiTNF- $\alpha$ agent, infliximab, was initiated. When the patient's treatment began, the tuberculin skin test was negative, chest radiography and interferon gamma release assay were normal, and no evidence of tuberculosis was found. After receiving the infliximab treatment (intravenous infliximab $5 \mathrm{mg} / \mathrm{kg}$ every 8 weeks), the patient began to experience symptom relief. Infliximab was injected 8 times, and the patients showed a very good response to the treatment, as his $\mathrm{CD}$ activity index indicated remission. At that time, the patient had a chest X-ray as part of regular outpatient follow-up. The identification of an abnormal pulmonary density was, therefore, an incidental observation. The patient 


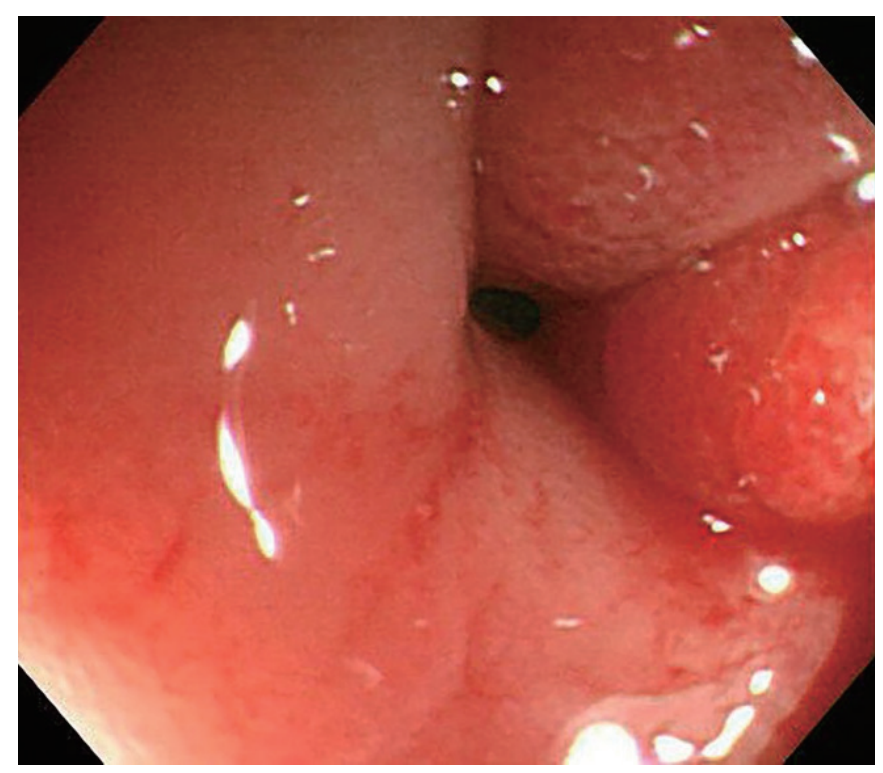

Fig. 1. Colonoscopy revealed strictures in the descending colon.

looked healthy and had no respiratory symptoms, such as cough or sputum. The tuberculin skin test was negative, and the interferon gamma release assay results were normal.

Chest X-ray and computed tomography displayed clustered small nodules in both lobes and multiple enlarged lymph nodes (Fig. 2). The results of a fungus culture, an acid-fast bacilli stain, a tuberculosis polymerase chain reaction (PCR) assay, and a bacterium culture in bronchial aspirate were all found to be negative. Endobronchial ultrasound-guided transbronchial needle aspiration of the subcarinal lymph node was performed, and histology demonstrated noncaseating granulomatous lesions (Fig. 3). Cancer and tuberculosis may, like sarcoidosis, accompany mediastinal lymphadenopathy. That possibility was examined by using acidfast staining, PCR, and cytology of the lymph node. All the results were negative. The serum angiotensin-converting enzyme level was normal (50 U/L; reference range, 20-70 U/L). At this stage, the diagnosis of sarcoidosis was made, and treatment with infliximab was discontinued. Lung lesions were improved 5 months after infliximab had been discontinued. Therefore, the anti-TNF treatment was thought to have triggered pulmonary sarcoidosis in this patient with IBD. Nevertheless, his paradoxical inflammations were not severe, and he wanted to continue anti-TNF- $\alpha$ therapy for his refractory $\mathrm{CD}$, so infliximab was continued cautiously. Subsequently, the patient's lung lesions spontaneously regressed to normal (Fig. 4). Since that time, pulmonary sarcoidosis has not recurred over 19 dosing periods spaced 8 weeks apart.

\section{DISCUSSION}

TNF- $\alpha$ is a cytokine that plays a prominent role in forming noncaseating granuloma in both sarcoidosis [2] and CD [3]. Inflix-
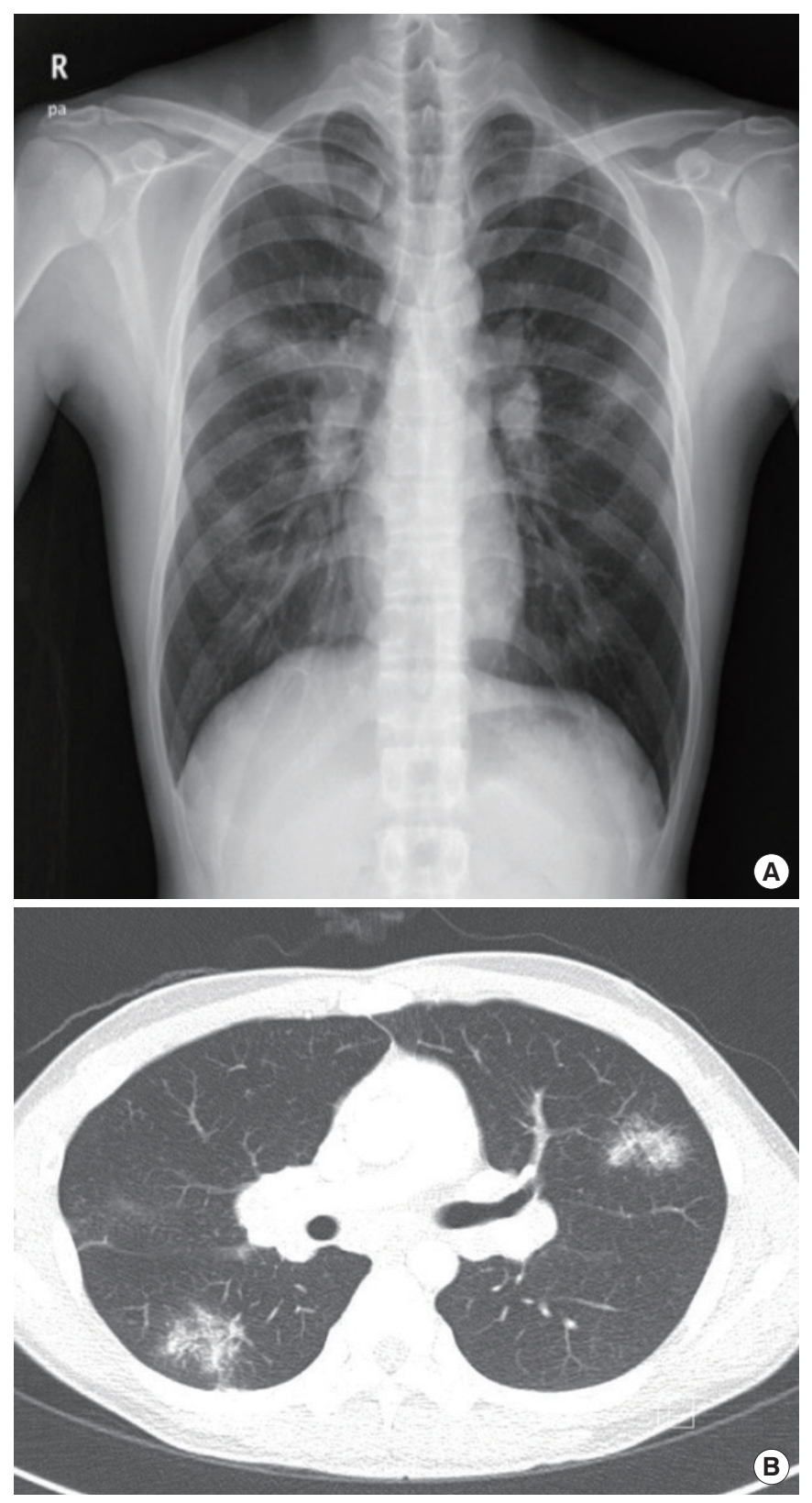

Fig. 2. (A) Chest X-ray showing prominent pulmonary hilar vessels and increased opacity in both upper lobes. (B) Chest computed tomography scan showing a cluster of centrilobular nodules in each lobe.

imab is administered to $\mathrm{CD}$ patients who have no reactions to steroids or immunosuppressive drugs. Also, additional evidence exists that infliximab is effective in patients with sarcoidosis who have had no positive reactions to steroids or general treatments [4]. Recently, anti-TNF- $\alpha$ therapy was reported to induce paradoxical inflammations, vasculitis, lupus, and sarcoidosis $[5,6]$. A clear mechanism for this paradoxical inflammation has yet to be identified; however, this paradoxical inflammation appears to be 


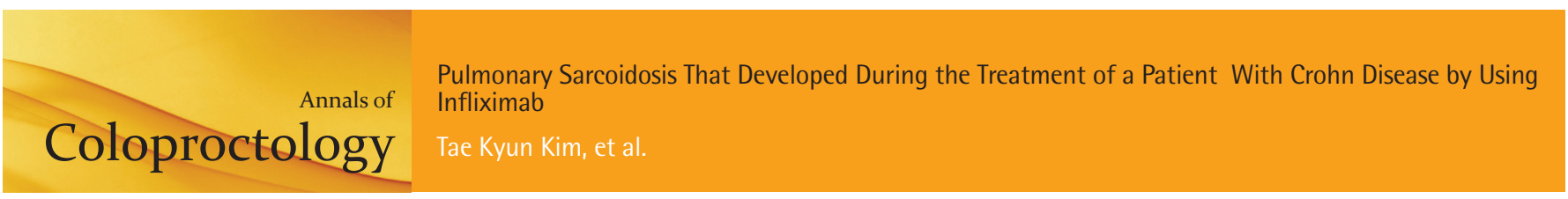

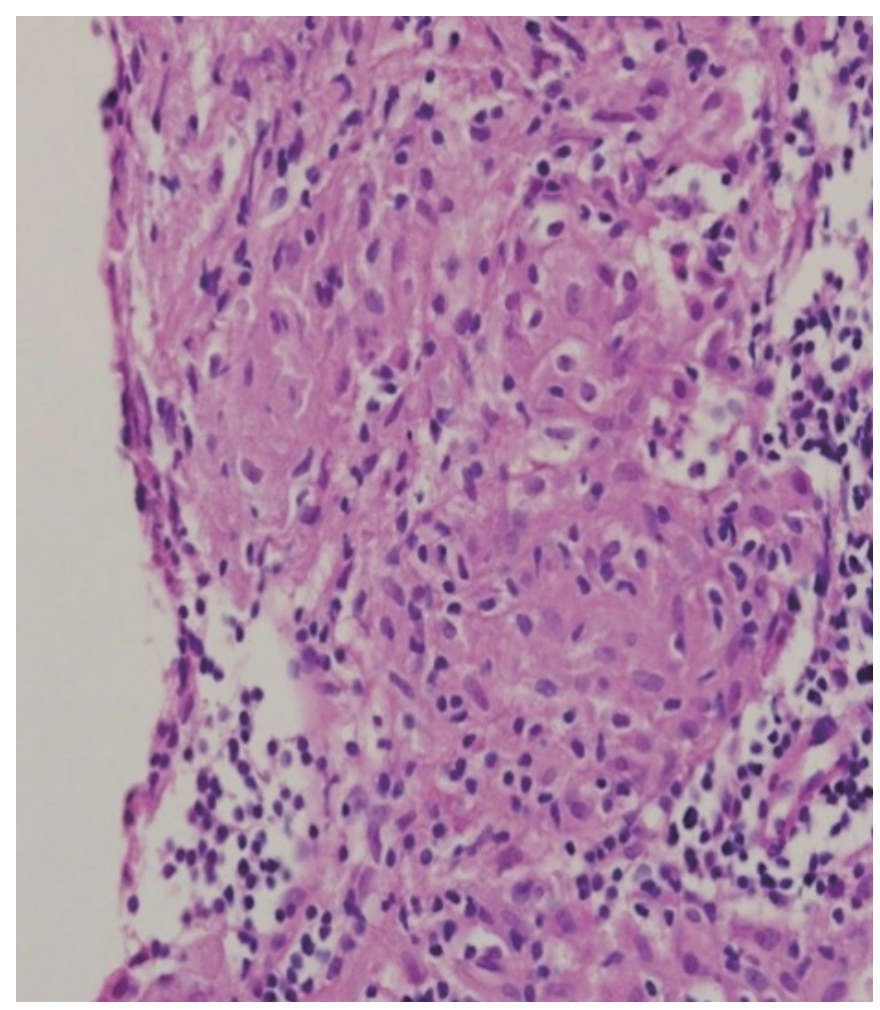

Fig. 3. Chronic granulomatous inflammation consisting mainly of epithelioid histiocytes and some lymphocytes, but no caseous necrosis $(\mathrm{H} \& \mathrm{E}, \times 400)$.

part of the underlying pathogenesis of this case report. To the best of our knowledge, a few cases of sarcoidosis occurring after the use of infliximab for $\mathrm{CD}$ have been reported overseas, but no Korean cases have been identified in the literature.

A foreign study published in 2010 reported on patients with autoimmune disease who were found to have sarcoidosis after having used anti-TNF- $\alpha$ agents. According to that research, out of 1,370 patients, 38 were found to have sarcoidosis after their use of anti-TNF- $\alpha$ agents $[7,8]$. Seventy-five percent of the cases used etanercept while $25 \%$ used infliximab. Most patients with sarcoidosis stopped anti-TNF- $\alpha$ treatment either because their sarcoidosis symptoms became severe or because they required treatment for tuberculosis or another respiratory infection. Nevertheless, a few patients who exhibited no infection and had good response to treatment with anti-TNF- $\alpha$ agents continued their therapy. Over time, the symptoms and the signs of sarcoidosis in the 2 groups spontaneously improved. Similarly, our patient's pulmonary sarcoidosis did not recur after reuse of infliximab. On resolution of sarcoidosis, a longer rechallenge interval with anti-TNF therapy was associated with a lower likelihood of recurrence on rechallenge [9]. Also, TNF- $\alpha$ genotypes and polymorphisms are known to be associated with sarcoidosis drug response [10]. In our opinion, specific TNF-a genotypes or other unknown factors might

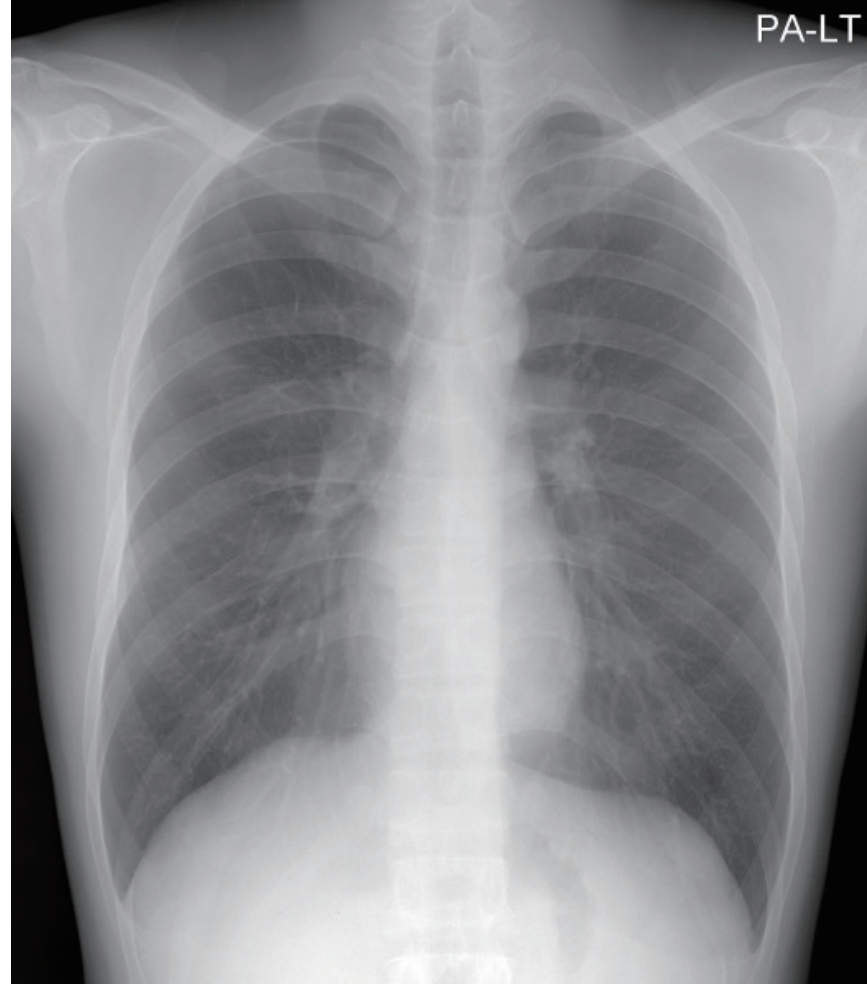

Fig. 4. The patient's lung lesions subsequently regressed to normal.

play a role in predicting the uneventful continuation of antiTNF- $\alpha$ therapy in this case.

As shown by the study results, determining whether drug treatment should continue or cease when side effects are experienced during use of anti-TNF agents is difficult. Therefore, we recommend that various aspects of the patient's health, including overall physical condition, the risk of inflammation, previous level of any anti-TNF agent reaction, and a patient's willingness to continue treatment, be taken into account. A literature review article [11] reported that cessation of anti-TNF- $\alpha$ agents resulted in spontaneous resolution of clinical symptoms and organ involvement (15 cases), improvement following corticosteroid treatment (30 cases), and persistence of sarcoidosis ( 7 cases). In our case, the patient had no symptoms and had experienced a negative reaction to treatments other than with anti-TNF agents. Therefore, after these factors and the patient's willingness to carry on with the treatment had been taken into account, infliximab treatment was continued. The patient's lung lesions improved spontaneously.

Few hypotheses can explain this paradox surounding treatment with infliximib. Lymphocytes or macrophages exhibit TNF- $\alpha$ on cell membranes or discharge them. Monoclonal antibodies like infliximab have high neutralizing potency toward membranous TNF- $\alpha$ and cause cell lysis by activating complements. Incomplete interruption promotes lymphocyte production of more cytokines for compensation [12]. Living lymphocytes and excess cytokines 
are thought to cause sarcoidosis. In summary, TNF- $\alpha$ inhibitors induce immunological disorder, thereby triggering sarcoidosis. As with other autoimmune diseases, complex interactions among genetic features, environmental factors, and immunological responses may serve to promote the development of sarcoidosis. In another theory, Eishi et al. [13] revealed the presence of Propionibacterium and mycobacterial DNA in the lymph nodes of patients with sarcoidosis; they suggested that infection was the cause of sarcoidosis. In 2012, van Moorsel and Christiani [14] stated that, according to a recent gene analysis, the mutations of certain genes caused both $\mathrm{CD}$ and sarcoidosis. In the clinical settings, determining whether sarcoidosis occurring after anti-TNF agent treatment represents true paradoxical inflammations, the intrathoracic presentation of $\mathrm{CD}$, or the coexistence of $\mathrm{CD}$ and sarcoidosis is extremely difficult.

In summary, our patient developed sarcoidosis after beginning infliximab treatment. Sarcoidosis has a variable clinical course, ranging from benign self-limited recovery to life-long disability. Because the patient did not have respiratory symptoms and opportunistic infections had been excluded, we did cease treatment and supportive care. Over time, the patient's condition improved spontaneously. In our case, determining whether sarcoidosis occurred after anti-TNF agent treatment, whether it was caused by a hidden infection, or whether a gene mutation induced both $\mathrm{CD}$ and sarcoidosi was difficults. If a patient is to be treated with antiTNF agents, unexpected diseases that may arise, such as sarcoidosis, must be taken into account, and regular follow-up examinations must be performed. If sarcoidosis occurs, a patient's overall physical condition and level of anti-TNF agent treatment reaction should be taken into careful consideration to determine whether to stop the treatment.

\section{CONFLICT OF INTEREST}

No potential conflict of interest relevant to this article was reported.

\section{REFERENCES}

1. Cathcart S, Sami N, Elewski B. Sarcoidosis as an adverse effect of tumor necrosis factor inhibitors. J Drugs Dermatol 2012;11:60912.
2. Iannuzzi MC, Rybicki BA, Teirstein AS. Sarcoidosis. N Engl J Med 2007;357:2153-65.

3. Abraham C, Cho JH. Inflammatory bowel disease. N Engl J Med 2009;361:2066-78.

4. Russell E, Luk F, Manocha S, Ho T, O'Connor C, Hussain H. Long term follow-up of infliximab efficacy in pulmonary and extrapulmonary sarcoidosis refractory to conventional therapy. Semin Arthritis Rheum 2013;43:119-24.

5. Borchers AT, Leibushor N, Cheema GS, Naguwa SM, Gershwin ME. Immune-mediated adverse effects of biologicals used in the treatment of rheumatic diseases. J Autoimmun 2011;37:273-88.

6. Vavricka SR, Wettstein T, Speich R, Gaspert A, Bachli EB. Pulmonary granulomas after tumour necrosis factor alpha antagonist therapy. Thorax 2003;58:278-9.

7. Ramos-Casals M, Brito-Zerón P, Muñoz S, Soto MJ; BIOGEAS STUDY Group. A systematic review of the off-label use of biological therapies in systemic autoimmune diseases. Medicine (Baltimore) 2008;87:345-64.

8. Ramos-Casals M, Roberto-Perez-Alvarez, Diaz-Lagares C, Cuadrado MJ, Khamashta MA; BIOGEAS Study Group. Autoimmune diseases induced by biological agents: a double-edged sword? Autoimmun Rev 2010;9:188-93.

9. van der Stoep D, Braunstahl GJ, van Zeben J, Wouters J. Sarcoidosis during anti-tumor necrosis factor-alpha therapy: no relapse after rechallenge. J Rheumatol 2009;36:2847-8.

10. Sharma S, Ghosh B, Sharma SK. Association of TNF polymorphisms with sarcoidosis, its prognosis and tumour necrosis factor (TNF)-alpha levels in Asian Indians. Clin Exp Immunol 2008; 151:251-9.

11. Sim JK, Lee SY, Shim JJ, Kang KH. Pulmonary sarcoidosis induced by adalimumab: a case report and literature review. Yonsei Med J 2016;57:272-3.

12. Dinarello CA. Differences between anti-tumor necrosis factor-alpha monoclonal antibodies and soluble TNF receptors in host defense impairment. J Rheumatol Suppl 2005;74:40-7.

13. Eishi Y, Suga M, Ishige I, Kobayashi D, Yamada T, Takemura T, et al. Quantitative analysis of mycobacterial and propionibacterial DNA in lymph nodes of Japanese and European patients with sarcoidosis. J Clin Microbiol 2002;40:198-204.

14. van Moorsel CH, Christiani DC. Genetic susceptibility to sarcoidosis, a chronic inflammatory disorder. Am J Respir Crit Care Med 2012;186:816-8. 